\title{
Hospital workforce mental reaction to the pandemic in a low COVID-19 burden setting: a cross-sectional clinical study
}

\author{
Panagiotis Alexopoulos ${ }^{1,2}$ (D) Dimitrios Roukas ${ }^{3} \cdot$ Apostolos Efkarpidis $^{4} \cdot$ Georgia Konstantopoulou ${ }^{5} \cdot$ \\ Rigas Soldatos ${ }^{6} \cdot$ Katerina Karaivazoglou $^{1} \cdot$ Evagellia Kontogianni ${ }^{6} \cdot K^{\prime}$ Konstantinos Assimakopoulos ${ }^{1}$. \\ Theodoros Iliou ${ }^{7} \cdot$ Polychronis Economou ${ }^{8} \cdot$ Philippos Gourzis $^{1} \cdot$ Antonios Politis $^{6,9}$
}

Received: 31 December 2020 / Accepted: 20 April 2021 / Published online: 27 April 2021

(c) Springer-Verlag GmbH Germany, part of Springer Nature 2021

\begin{abstract}
TheCOVID-19 pandemic has mental health implications for both healthcare workforces and general population, particularly in regions heavily hit by the crisis. The study aimed (i) to investigate anxiety- and depression severity differences between staff of a COVID-19 treatment unit $(N=84)$ and a hospital without such a unit $(N=55)$ in comparison to participants of a convenience general population online survey $(N=240)$ and (ii) to explore relations between such symptoms and hospital staff reaction to COVID-19 in a low COVID-19 burden setting. Anxiety was studied with the Generalized Anxiety Disorder 7-Item in hospital workforces and with the Hospital Anxiety Depression Scale (HADS) in online survey participants. Depression symptoms were assessed with the Patient Health Questionnaire-9 in hospital employees and the HADS in the online survey sample. Symptoms were classified as absent/minimal, borderline abnormal or indicating clinical caseness. Staff reaction to COVID-19 was tapped with a 9-item-questionnaire and the 22-item Impact of Event Scale-revised (IES-R). Proper tests for differences and stepwise ordered logistic regression models were employed. Anxiety- and depression severity was higher in hospital workforces than in online survey participants $(P<0.05)$. Anxiety was more severe in frontline- compared to backstage employees $(P<0.001)$ was inversely correlated with age $(P=0.011)$ and positively with avoidance $(P=0.028)$. Both anxiety and depression symptoms related to intrusion symptoms $(P<0.001)$. Regarding the relatively long data collection period, an inverse association between crisis duration and depression symptoms was detected $(P=0.025)$. These observations point to the urgent need for distress-mitigating interventions for hospital workforces even in low COVID-19 burden settings.
\end{abstract}

Keywords Mental impact of COVID-19 crisis · Anxiety · Depression · Preventive behaviours · Worries

Panagiotis Alexopoulos

panos.alexopoulos@upatras.gr

1 Department of Psychiatry, Faculty of Medicine, School of Health Sciences, Patras University General Hospital, University of Patras, Patras, Greece

2 Department of Psychiatry and Psychotherapy, Klinikum Rechts Der Isar, Faculty of Medicine, Technische Universität München, Munich, Germany

3 Department of Psychiatry, 417 Army Equity Fund Hospital (NIMTS) Hospital, Athens, Greece

4 Nursing Services Department, General Hospital of Syros "Vardakeio and Proio", Ermoupolis, Greece

5 Special Office for Health Consulting Services and Faculty of Education and Social Work, School of Humanities and Social Sciences, University of Patras, Patras, Greece
6 First Department of Psychiatry, School of Medicine, Eginition Hospital, National and Kapodistrian University of Athens, Athens, Greece

7 Medical Informatics Laboratory, Faculty of Medicine, School of Health Sciences, Democritus University of Thrace, Alexandroupolis, Greece

8 Department of Civil Engineering (Statistics), University of Patras, Patras, Greece

9 Division of Geriatric Psychiatry and Neuropsychiatry, Department of Psychiatry, Johns Hopkins Medical School, Baltimore, MD, USA 


\section{Introduction}

The clinical consequences of the new virus SARS-CoV-2 (COVID-19) range from asymptomatic phenotypes to acute respiratory distress syndrome, metabolic acidosis, liver, kidney and heart failure, but has also implications for mental health [1-3]. These clinical uncertainties in conjunction with the socioeconomic effects of draconic measures (e.g. confinement, quarantine) aiming to contain the COVID-19 spread were shown to pertain to psychological distress, anxiety and mood disturbances [4-6] or even to symptoms of posttraumatic stress disorder, since the pandemic outbreak of infections the outcome of which is hardly predictable can be perceived as a traumatic experience $[7,8]$.

Health care professionals stand in the epicentre of the COVID-19 crisis management and bear an extraordinarily heavy burden. Particularly medical and paramedical staff working at COVID-19 units and hospital intensive care units has been confronted during the pandemic with physical and mental exhaustion [9-11]. Working and decision making in hospital settings with overwhelming influx of patients suffering from potentially life-threatening infections and with limited available ventilators, personal protective equipment and lifesaving medications embody a physical, mental and occasionally a moral challenge [12, 13]. Moreover, the spread of COVID-19 among hospital workforces results in the physical exhaustion due to work overload of those remaining on the frontline and keeping the flag flying, despite being stigmatized in many cases as vectors of contagion [14, 15]. Studies report a high prevalence of anxiety and depressive symptoms, emotional exhaustion and insomnia in healthcare professionals working in the first line of care [9, 16-22]. Of note, these data are derived in most cases from observations in regions which have been heavily hit by the pandemic and/or at hospitals caring for COVID-19 patients.

The current study aimed to provide pieces of evidence on the mental effects of the COVID-19 crisis on hospital workforces in Greece, where the COVID-19 burden [23] was low in comparison to other countries during the first COVID-19 surge [24, 25]. It aimed to explore differences in anxiety and depression symptom severity between COVID-19 frontline- and backstage hospital employees compared to a convenience sample of individuals without reported mental disorders, as well as to investigate relations between such symptoms and demographic data, restrictive measures and worries, mental and behavioural reaction of hospital staff to the COVID-19 crisis. It was hypothesized that even in a such a low burden setting (i) depression and anxiety symptom severity would have been higher in hospital employees, particularly in frontline workforces, in comparison to the convenience sample and that (ii) mental and behavioural reaction of hospital staff to the COVID-19 crisis would have pertained to anxiety and depression symptom severity.

\section{Methods}

\section{Participants}

The dataset of hospital workforces was collected between June 1st and July 19th 2020 and included staff of the COVID-19 treatment unit of the 417 Army Equity Fund (NIMTS) Hospital in Athens, Nomenclature of Territorial Units for Statistics 2 (NUTS 2) region of Attica, and medical and paramedical employees of the General Hospital of Syros "Vardakeio and Proio", NUTS 2 region of South Aegean, a hospital without a COVID-19 treatment unit. To enrich study design, the demographic data and anxiety and depression symptoms of hospital workforces were compared to data of residents of the regions of Attica and South Aegean without mental disorders who were derived from an online survey in a convenience sample of the general population in Greece. The online survey was conducted during the first COVID-19 confinement period [26] between April 11th and May 1st 2020 and was a wide-scale, anonymous study, based on a questionnaire which was prepared in an online format with Google Form and was distributed through social media and a number of press releases [26]. Inclusion criteria of the present study were (i) being 18 years old or older, (ii) working at the COVID-19 unit of the 417 Army Equity Fund Hospital in Athens or at the General Hospital of Syros "Vardakeio and Proio", or online survey participation and (iii) residency in the NUTS 2 regions of Attica or South Aegean, where the two study hospitals are situated. Exclusion criteria included: (i) incomplete or missing responses except for missing data regarding education, (ii) COVID-19 infection or flu-like self-limiting symptoms, (iii) being on leave due to health problems, (iv) current acute/unstable phase of a mental disorder and additionally for online survey participants previous mental health problems which are strong predictors of anxiety and depression in general population during the COVID-19 crisis [5] and (v) residency in NUTS 2 regions other than Attica or South Aegean. The study was conducted in accordance with the latest revision of the Declaration of Helsinki. Dataset collection and study aims were approved by the ethics committees of the Kapodistrian and National University of Athens and the Patras University General Hospital. Informed consent was obtained from all participants after a thorough description of data dataset collection procedures and aims. 


\section{Procedures}

The assessment of study participants was based on valid and well-established instruments capturing depression and anxiety symptoms, whilst the behavioural and mental reaction of hospital workforces to the COVID-19 crisis was tapped with a standardized set of questions as well as with the 22-item Impact of Event Scale-revised (IES-R) [27].

\section{Depression symptoms}

Depression symptoms were assessed with the Patient Health Questionnaire-9 (PHQ-9) in hospital workforces and with the depression subscale of the Hospital Anxiety and Depression Scale (HADS-D) in the general population online survey participants. PHQ-9 is a self-report tool for depression detection [28-30]. The nine items of the tool are scored from 0 ("not at all") to 3 points ("almost every day") and the total severity score can range from 0 to 27 points. Total scores between 0 and 4 indicate no depression symptoms, scores between 5 and 9 points indicate borderline symptoms, whilst higher scores are compatible with clinical depressive syndromes (e.g. minor and major depression, dysthymia) [31]. HADS-D is an another self-rating tool capturing depression symptoms [32, 33]. It consists of seven items, which can be answered with four ordinal choices ranging between 0 and 3. The HADS-D total score ranges from 0 to 21 . Individuals with 0 to 7 points are classified as normal, with 8 to 10 points as borderline abnormal and with scores higher than 10 as suffering from a clinical depressive syndrome. Based on the aforementioned cut-off points, study participants were classified with regard to depression symptoms as having minimal or no depression symptoms, as borderline abnormal or as clinical cases.

\section{Anxiety symptoms}

Anxiety symptoms were tapped with the Generalized Anxiety Disorder 7-Item (GAD-7) in hospital staff and the HADS anxiety subscale (HADS-A) in the general population online survey participants. The well-established GAD-7, which was initially developed for the assessment of generalized anxiety disorder, is a useful self-report anxiety severity scale [34]. It is composed of seven items, each of which is scored based on a four-point severity scale ranging from 0 indicating "not at all" to 4 indicating for instance "nearly every day", "very definitely" or "quite badly" [35]. Total scores range between 0 and 21 . Very mild symptoms are compatible with $0-5$ points, scores between 6 and 10 point to borderline abnormal cases, whilst higher scores indicate a clinically significant condition [34]. HADS-A consists of seven intermingled items, too $[32,33]$. They can be answered with four ordinal choices (0-3). The HADS-A total score ranges between 0 and 21. Participants with $0-7$ points are classified as normal, with 8-10 points as borderline abnormal, whilst scores higher than 10 indicate a clinical anxiety syndrome [32,33]. Again, based on the aforementioned cut-offs, study participants were classified with regard to anxiety symptoms into three categories: minimal or no anxiety symptoms, bordeline abnormal or into the category of clinical cases.

\section{COVID-19 crisis related behaviours, attitudes and mental reaction}

Relying on previous observations referring to settings with heavy COVID-19 burden [9, 16, 22, 36, 37], seven questions about preventive behaviours towards COVID-19 and four questions about COVID-19 infection worries were posed to hospital workforces (Table 1). In both cases, compositive scores were calculated. To assess trust in safety and COVID-19 management at workplace five items were employed. Six additional items captured workplace related worries (Table1). Except for the items on preventive behaviours towards COVID-19, which were measured on a fivepoint Likert scale $(0-4$, with 0 meaning "not at all" and 4 meaning "always"), items were rated on four-point Likert scale in all other cases (with 0 meaning "not at all" and 3 "much"). The internal consistency of the items which were employed to assess preventive behaviours towards COVID19 (seven items), trust in safety and COVID-19 management at workplace (five items) and COVID-19 infection related worries (four items) was good to excellent (Cronbach alpha correlation coefficients were $0.63,0.74,0.84$, respectively). In addition, the 22-item Impact of Event Scale-revised (IESR) [27] was used. Respondents were asked to indicate how much they were distressed or bothered during the past week by each "difficulty" listed in regard to the epidemic outbreak. Each item is rated on a five-point scale ranging from 0 ("not at all") to 4 ("extremely"). The IES-R assesses avoidance (the tendency to avoid thoughts or reminders of the pandemic), intrusion (difficulties in staying asleep, dissociative experiences similar to flashbacks) and hyperarousal (irritated feeling, difficulties in sleep onset) [27].

\section{COVID-19 crisis parameters}

The first confirmed case of COVID-19 in Greece was reported on February 26th, 2020. In response to increasing numbers of cases vigorous confinement measures were imposed from March 23rd to May 4th, which were relaxed afterwards. Based on data of the Oxford COVID-19 Government Response Tracker (OxCGRT) [24], the government stringency index was taken into account in the analyses. It is a composite measure of nine response metrics, i.e. school closures; workplace closures; cancellation of public events; restrictions on public gatherings; closures of public 
Table 1 Items capturing COVID-19 crisis related behaviours, attitudes and worries

\author{
Preventive behaviours towards COVID-19 \\ 1. When I cough or sneeze, I do so in the bend of my elbow or I use a tissue \\ 2. After contact to patient, I wash my hands with soap and water thoroughly or use hand sanitizer \\ 3. After coughing or sneezing, I wash my hands with soap and water thoroughly or use hand sanitizer \\ 4. At work I wear a mask \\ 5. At work I keep the necessary distance of $2 \mathrm{~m}$ from colleagues \\ 6. I avoid crowded places \\ 7. I try to keep the necessary distance of $2 \mathrm{~m}$ from patients, if possible \\ Trust in safety and COVID-19 management at workplace \\ 1. Satisfaction with availability of personal safety equipment \\ 2. Trust in the capacity of the hospital medical staff to diagnose COVID-19 \\ 3. Feelings of better protection at workplace than elsewhere \\ 4. Satisfaction with solidarity at workplace \\ 5. Satisfaction with updates on COVID-19 crisis at workplace \\ Worries about job loss due to COVID-19 infection \\ Uncertainty feelings at workplace due to COVID-19 pandemic \\ Self-confidence at work \\ Positive thinking regarding occupational difficulties \\ Feeling supported by family \\ Support to colleagues \\ COVID-19 infection related worries \\ 1. Worries to be infected with COVID-19 \\ 2. Worries to pass away due to COVID-19 \\ 3. Worries to be vector of disease transmission \\ 4. Worries regarding possible COVID-19 infection of family members and close friends
}

transport; stay-at-home requirements; public information campaigns; restrictions on internal movements and international travel controls. The index on any given day is calculated as the mean score of the nine metrics, each taking a value between 0 and 100 . Higher scores mirror stricter government responses (i.e. $100=$ strictest response). If policies vary at the subnational level, the index is shown as the response level of the strictest sub-region.

\section{Statistical analyses}

Differences in anxiety- and depression symptom severity, sex-, age category and educational level distribution between groups were explored with the Pearson's chi-squared test. The Mann-Whitney test and the Kruskal-Wallis test were employed, as appropriate, for assessing further differences between the groups, since data normality assumption was rejected based on the Shapiro-Wilk test for normality. Stepwise ordered logistic regression models (alpha to enter $=0.10$, alpha to remove $=0.10$ ) was employed for studying the impact of demographic and occupational data and reaction to COVID-19 pandemic outbreak on anxietyand depression symptom severity of frontline and backstage hospital workforces. All results with $P<0.05$ were deemed statistically significant.

\section{Results}

From the members of the medical- $(N=40)$ and nursing $(N=100)$ staff of the COVID-19 treatment unit and from the backstage hospital staff (45 physicians, 183 paramedical staff [nursing staff, occupational therapists, physiotherapists, medical laboratory technologists, midwives] and 65 administrative staff) who were potentially eligible for study enrollment, 106 frontline- and 108 backstage employees consented to study participation. Unfortunately, in questionnaires of 22 COVID-19 frontline- and 53 backstage hospital employees and 13 participants of the general population online survey, there were items which were not answered and total scores were not calculated. Thus, these cases were not considered in the analyses. Of note, no differences were detected in the distribution either of age- and education categories or sex between individuals included in the analyses and dropouts due to incomplete dataset, in any of the study groups (in all cases $P>0.05$ ). The COVID-19 frontline hospital group included 22 members of medical and 62 members of nursing staff; the backstage group consisted of 5 physicians, 29 paramedical professionals and 21 hospital administrative employees, whilst the online survey group encompasssed 208 adults who lived in the NUTS1 region of Attica and 32 living in the South Aegean region 
(Table 2). Both anxiety and depression symptom severity significantly differed between the study groups (Pearson chisquared $=117.94, P=2 \cdot 10^{-24}$ and $15.57,0.004$, respectively) (Table 3). More specifically, hospital workforces manifest more severe depression- and anxiety symptoms compared to online survey participants. Anxiety symptoms became more severe across the study groups as exposure to COVID19 cases increased and reached their maximum in frontline hospital employees. Of note, all COVID-19 frontline hospital employees reported at least very mild anxiety symptoms. In contrast, depression symptoms were more severe in backstage employees compared to both frontline workforces and online survey participants (Table 3). Interestingly, more than $50 \%$ of backstage workforces experienced at least mild depression symptoms. The time since the diagnosis of the first COVID-19 case in Greece and study participation and the stringency index at timepoint of study participation varied across the groups (in both cases $P=0.0001$ ) (Table 2). Except for differences in trust in safety and COVID-19 management at workplace, COVID-19 infection related worries and positive thinking regarding occupational difficulties during the pandemic, no further differences were detected between frontline and backstage hospital workforces with regard to parameters of reaction to the COVID-19 crisis (Table 3). It is noteworthy that uncertainty feelings at workplace due to COVID-19 pandemic in frontline professionals

Table 2 Sociodemographic characteristics of the study sample

\begin{tabular}{|c|c|c|c|c|c|c|}
\hline & \multirow{2}{*}{$\begin{array}{l}\text { General population } \\
\text { online survey participants } \\
\text { (Group } 1, \mathrm{G} 1 \text { ) }\end{array}$} & \multirow{2}{*}{$\begin{array}{l}\text { COVID-19 backstage } \\
\text { hospital workforce } \\
\text { (Group 2, G2) }\end{array}$} & \multirow{2}{*}{$\begin{array}{l}\text { COVID-19 frontline hos- } \\
\text { pital workforce (Group } \\
3, \text { G3) }\end{array}$} & \multicolumn{3}{|c|}{ Pairwise comparisons } \\
\hline & & & & G1 vs. G2 & G1 vs. G3 & $\mathrm{G} 2$ vs. G3 \\
\hline$N$ overall & 240 & 55 & 84 & & & \\
\hline $\begin{array}{l}\text { Age groups, years, }(N \\
[\%])\end{array}$ & \multicolumn{3}{|c|}{ Overall: Pearson $\chi^{2}(10) 36.76 P<0.001$} & $P<0.001$ & $P=0.370$ & $P=0.001$ \\
\hline $18-30$ & 88 [37] & $4[7]$ & $27[32]$ & & & \\
\hline $31-40$ & $45[19]$ & $10[18]$ & $21[25]$ & & & \\
\hline $41-50$ & $58[24]$ & $30[55]$ & $20[24]$ & & & \\
\hline $51-60$ & $34[14]$ & $11[20]$ & $15[18]$ & & & \\
\hline $61-70$ & $13[5]$ & $0[0]$ & $1[1]$ & & & \\
\hline $70+$ & $2[1]$ & $0[0]$ & $0[0]$ & & & \\
\hline Age (in years) ${ }^{\mathrm{a}}$ & & $43.98(7.90)[25-59]$ & $39.0(10.8)[23-62]$ & & & $P=0.006$ \\
\hline \multirow[t]{2}{*}{ Sex (female, $N[\%]$ ) } & \multicolumn{3}{|c|}{ Overall: Pearson $\chi^{2}(2) 4.03 P=0.133$} & $P=0.096$ & $P=0.156$ & $P=0.666$ \\
\hline & $160[67]$ & $43[78]$ & $63[75]$ & & & \\
\hline Education level ( $N[\%])$ & \multicolumn{3}{|c|}{ Overall: Pearson $\chi^{2}$ (6) $46.70 P<0.001$} & $P<0.001$ & $P<0.001$ & $P<0.001$ \\
\hline Primary education & $0[0]$ & $0[0]$ & $4[7]$ & & & \\
\hline Secondary education & $69[29]$ & $31[56]$ & 9 [15] & & & \\
\hline $\begin{array}{l}\text { First cycle university } \\
\text { studies }\end{array}$ & $106[44]$ & $19[35]$ & $24[41]$ & & & \\
\hline Postgraduate studies & 75 [27] & $5[9]$ & $22[37]$ & & & \\
\hline \multicolumn{7}{|l|}{ Profession $(N[\%])$} \\
\hline Medical staff & & $5[9]$ & $22[26]$ & & & $P<0.001$ \\
\hline Paramedical staff & & $29[53]$ & $62[74]$ & & & \\
\hline Administrative staff & & $21[38]$ & $0[0]$ & & & \\
\hline \multirow{2}{*}{$\begin{array}{l}\text { Time since the diagno- } \\
\text { sis of the first COVID- } \\
19 \text { case in Greece (in } \\
\text { days) }\end{array}$} & \multicolumn{2}{|l|}{ Overall: $276.40 P<0.001$} & & $P<0.001$ & $P<0.001$ & $P<0.001$ \\
\hline & $51.02(5.45)[44-64]$ & 100.96 (4.51) [95-109] & $118.61(8.6)[109-134]$ & & & \\
\hline \multirow[t]{2}{*}{ Stringency index ${ }^{a}$} & \multicolumn{2}{|l|}{ Overall: $274.16 \mathrm{P}<0.001$} & & $P<0.001$ & $P<0.001$ & $P<0.001$ \\
\hline & $84.26(0)[84.26-84.26]$ & $\begin{array}{l}55.71(2.64)[54.63- \\
62.04]\end{array}$ & $\begin{array}{l}44.25(4.61)[40.74- \\
54.63]\end{array}$ & & & \\
\hline $\begin{array}{l}\text { Contact to COVID-19 } \\
\text { cases }(N[\%])\end{array}$ & & $12[22]$ & $84[100]$ & & & $P<0.001$ \\
\hline
\end{tabular}

All comparisons are based on Kruskal-Wallis test and Mann-Whitney $U$ test, as appropriate for continuous data, or chi-squared test for categorical data (homogeneity hypothesis)

$G$ group

${ }^{a}$ Data presented as mean (standard deviation) [minimum-maximum] 
Table 3 Anxiety-, depression- and COVID-19 reaction data of the study sample

\begin{tabular}{|c|c|c|c|c|c|c|}
\hline & \multirow{2}{*}{$\begin{array}{l}\text { General population } \\
\text { online survey participants } \\
\text { (Group } 1, \mathrm{G} 1 \text { ) }\end{array}$} & \multirow{2}{*}{$\begin{array}{l}\text { COVID-19 backstage } \\
\text { hospital workforce } \\
\text { (Group 2, G2) }\end{array}$} & \multirow{2}{*}{$\begin{array}{l}\text { COVID-19 frontline hos- } \\
\text { pital workforce (Group } \\
3, \text { G3) }\end{array}$} & \multicolumn{3}{|c|}{ Pairwise comparisons } \\
\hline & & & & G1 vs. G2 & G1 vs. G3 & $\mathrm{G} 2$ vs. G3 \\
\hline $\begin{array}{l}\text { Anxiety symptom sever- } \\
\text { ity }(N[\%])\end{array}$ & \multicolumn{3}{|c|}{ Overall: Pearson $\chi^{2}(4)=117.93 P=2 \cdot 10^{-24}$} & $P<0.001$ & $P<0.001$ & $P<0.001$ \\
\hline Absent or minimal & $163[68]$ & $21[38]$ & $0[0]$ & & & \\
\hline Borderline abnormal & 45 [19] & $19[35]$ & $46[55]$ & & & \\
\hline Clinical case & $32[13]$ & $15[27]$ & $38[45]$ & & & \\
\hline $\begin{array}{l}\text { Depression symptom } \\
\text { severity }(N[\%])\end{array}$ & \multicolumn{2}{|c|}{ Overall: Pearson $\chi^{2}(4)=15.57 \mathrm{P}=0.004$} & & $P<0.001$ & $P=0.572$ & $P=0.040$ \\
\hline Absent or minimal & $177[74]$ & $26[47]$ & $57[68]$ & & & \\
\hline Borderline abnormal & $41[17]$ & $21[38]$ & $17[20]$ & & & \\
\hline Clinical case & $22[9]$ & $8[15]$ & $10[12]$ & & & \\
\hline \multicolumn{7}{|c|}{ Reaction to COVID-19 crisis } \\
\hline \multicolumn{2}{|l|}{$\begin{array}{l}\text { Preventive behaviours } \\
\text { and attitudes towards } \\
\text { COVID }-19^{\mathrm{a}}\end{array}$} & $22.36(3.30)[16-28]$ & $22.33(3.74)[14-28]$ & & & $P=0.821$ \\
\hline \multicolumn{2}{|l|}{$\begin{array}{l}\text { Trust in safety and } \\
\text { COVID-19 manage- } \\
\text { ment at workplace }{ }^{\mathrm{a}}\end{array}$} & 9.53 (3.16) [2-15] & $10.89(2.69)[3-15]$ & & & $P=0.008$ \\
\hline \multicolumn{2}{|l|}{$\begin{array}{l}\text { Worries related to } \\
\text { COVID-19 infection }\end{array}$} & 7.85 (3.14) [0-12] & $5.81(3.42)[0-12]$ & & & $P<0.001$ \\
\hline \multicolumn{2}{|l|}{$\begin{array}{l}\text { Worries about job loss } \\
\text { due to COVID-19 } \\
\text { infection }^{\mathrm{a}}\end{array}$} & $0.45(0.86)[0-3]$ & $0.35(0.7)[0-3]$ & & & $P=0.664$ \\
\hline \multicolumn{2}{|l|}{$\begin{array}{l}\text { Uncertainty feelings } \\
\text { at workplace due to } \\
\text { COVID-19 pandemic }\end{array}$} & $1.25(0.93)[0-3]$ & $1.57(1.0)[0-3]$ & & & $P=0.069$ \\
\hline \multicolumn{2}{|l|}{$\begin{array}{l}\text { Positive thinking } \\
\text { regarding occupa- } \\
\text { tional difficulties } \\
\text { during the COVID-19 } \\
\text { pandemic }^{\text {a }}\end{array}$} & $2.49(0.57)[1-3]$ & $2.73(0.53)[1-3]$ & & & $P=0.024$ \\
\hline \multicolumn{2}{|l|}{$\begin{array}{l}\text { Self-confidence at work } \\
\text { during COVID-19 } \\
\text { crisis }^{\mathrm{a}}\end{array}$} & $0.69(0.96)[0-3]$ & $0.74(0.92)[0-3]$ & & & $P=0.684$ \\
\hline \multicolumn{2}{|l|}{$\begin{array}{l}\text { Support by family dur- } \\
\text { ing COVID-19 } \text { crisis }^{\text {a }}\end{array}$} & $0.73(0.91)[0-3]$ & $0.5(0.78)[0-3]$ & & & $P=0.200$ \\
\hline \multicolumn{2}{|l|}{$\begin{array}{l}\text { Support to colleagues } \\
\text { during COVID-19 } \\
\text { crisis }^{\mathrm{a}}\end{array}$} & $2.42(0.74)[0-3]$ & $2.60(0.58)[0-3]$ & & & $P=0.235$ \\
\hline \multicolumn{7}{|c|}{ Impact of event scale-revised (IES-R) } \\
\hline \multicolumn{2}{|c|}{ Intrusion $^{\mathrm{a}}$} & $0.78(0.82)[0-3.63]$ & $0.66(0.76)[0-3.63]$ & & & $P=0.391$ \\
\hline \multicolumn{2}{|l|}{ Avoidance $^{\mathrm{a}}$} & $1.04(0.70)[0-2.75]$ & $0.83(0.66)[0-2.75]$ & & & $P=0.084$ \\
\hline \multicolumn{2}{|l|}{ Hyperarousal $^{\mathrm{a}}$} & $0.88(0.74)[0-3]$ & $0.84(0.77)[03.5]$ & & & $P=0.620$ \\
\hline
\end{tabular}

All comparisons are based on Kruskal-Wallis test and Mann-Whitney $U$ test, as appropriate for continuous data, or chi-squared test for categorical data (homogeneity hypothesis)

${ }^{a}$ Data presented as mean (standard deviation) [minimum-maximum]

tended to exceed those of backstage hospital employees $(P=0.069)$.

The first stepwise ordered logistic regression model included anxiety symptom severity as dependent variable and depression symptom severity was the dependent variable in the second regression model. Demographic and occupational data (except for education level due to missing data) and parameters depicting reaction to the COVID19 pandemic outbreak presented in Tables 2 and 3 were included in both models as independent variables (19 variables in total). The final selected model (Table 4) revealed that intrusion and avoidance symptoms, age, support by 
Table 4 Impact (odds ratio) of demographic and occupational data and of data related to COVID-19 reaction on anxiety and depression symptom severity of hospital workforces according to the stepwise ordered logistic regression models

\begin{tabular}{lll}
\hline & Ordered depended variable & \\
\cline { 2 - 3 } & Anxiety symptom severity & Depression symptom severity \\
\hline Backstage vs. frontline hospital workforces (site) & $4.67\left(P<0.001^{* * *}\right)$ & $0.30\left(P=0.011^{*}\right)$ \\
Age group & $2.35\left(P=0.028^{*}\right)$ & $1.54(P=0.052)$ \\
Avoidance & $1.78\left(P=0.048^{*}\right)$ & $7.16\left(P<0.001^{* * *}\right)$ \\
Support by family during COVID-19 crisis & $14.51\left(P<0.001^{* * *}\right)$ & $0.84\left(P=0.002^{* *}\right)$ \\
Intrusion & & $0.96\left(P=0.025^{*}\right)$ \\
Preventive behaviours and attitudes towards COVID-19 & & $0.89\left(P=0.074^{*}\right)$ \\
Time since the diagnosis of the first COVID-19 case in Greece (in & & Pseudo $R^{2}=0.268$ \\
$\quad$ days) & & LR $\chi^{2}(5)=69.08, P=1.6 \cdot 10^{-13}$ \\
Worries related to COVID-19 infection & Pseudo $R^{2}=0.407$ & LR $\chi^{2}(5)=113.97, P=6 \cdot 10^{-23}$ \\
\end{tabular}

An odds ratio higher than 1 indicates a positive association, whilst a value lower than 1 points to an inverse association

*Significant at 0.05 level, **significant at 0.01 level, ***significant at 0.001 level

family and working at a COVID-19 unit compared to being tasked with other duties pertained to anxiety levels independently of profession. Depression levels were significantly related to intrusion symptoms, personal protection measures, support by family and crisis duration, whilst worries related to COVID-19 tended to affect depression symptoms according to the final selected model with depression symptom severity as dependent variable (Table 4).

\section{Discussion}

Casting light on depression and anxiety symptoms of hospital workforces compared to general population during the COVID-19 crisis, even in regions with low COVID19 burden, is important, since such forces form one of the backbones of the crisis management worldwide. Mapping the effects of their behavioural and mental reaction to the COVID-19 crisis on depression and anxiety symptoms can provide a solid basis of evidence and reason, icily dictating health policy choices aiming to accelerate creation of distress-mitigating interventions and protect mental balance and quality of life of people working at the epicentre of the pandemic management [19]. The present study was conducted during the first pandemic surge in Greece, which did not lead to an overwhelming COVID-19 burden; the study was not restricted to hospital employees tasked to treat COVID-19 cases and considered the intensity of pandemic containment measures. In line with previous reports, anxiety severity increased from general population online survey participants to hospital workforces and reached its maximum in COVID-19 frontline workforces, since these employees are more intensively exposed to demanding and unforeseen medical emergencies, (moral) trauma experiences $[8,38]$ and to high risk for becoming infected and transmitting COVID-19 to their social network compared to the other study groups $[4,7,16,17,19,21,22,39]$. It is noteworthy that the results of the ordered logistic regression model with anxiety symptom severity as dependent variable provided further evidence for the significant differences in anxiety symptoms between COVID-19 frontline- and backstage hospital employees (Table 4).

Despite the considerable symptom overlap between anxiety and depression and their common pathogenetic underpinnings [40], there was a decoupling between the pattern of anxiety and depression symptom variation across the groups. In accordance with the pattern of anxiety symptom variation, more severe depression symptoms were detected in backstage hospital workforces compared to online survey participants, while quite unexpectedly, frontline health professionals reported less severe depression symptoms than backstage hospital employees. This difference between frontline- and backstage hospital staff should be treated with caution especially in the light of the lack of significant association between depression symptom severity and serving as COVID-19 frontline- or backstage hospital employee (Table 4). It warrants further investigation and needs replication before final conclusions can be drawn. Interestingly, fewer symptoms pointing to depression such as appetite and sleep disturbances, fatigue, numbness, irritabillity were reported in COVID-19 frontline nurses than backstage nurses and general population during the COVID-19 crisis in China [32]. Thus, it can be reckoned that despite high anxiety levels and uncertainty feelings related to exposure to COVID-19 cases at workplace, at least some frontline health professionals exhibit unique mental and physical 
relisience and endurance and do not develop mood disturbances, despite the severity of their anxiety symptoms. The detected significantly less worries related to COVID-19 infection, the higher levels of positive thinking regarding occupational difficulties and the observed higher degree of trust in safety and COVID-19 management at workplace in frontline- than backstage staff (Table 3) may point to this unique mental and physical resilience and endurance of at least some COVID-19 frontline health professions.

Anxiety levels in hospital workforces were found to be positively related to intrusion and avoidance symptoms and support by family; age was inversely associated with anxiety, whilst COVID-19 frontline workforces suffered from more severe anxiety symptoms compared to backstage workforces. Taking into account that intrusion- and avoidance symptoms embody reactions to calamitous life events [27], the positive association between these symptoms and anxiety highlights, in accordance with previous reports [4, 39], the linkage between anxiety symptoms and mental reaction of hospital employees to the COVID-19 pandemic adversities even in a setting not heavily burdened by the crisis. Factors such as limited access to personal protective equipment or satisfaction with solidarity at workplace, which had been previously shown to affect anxiety levels [19], were not found here to exert a significant influence on anxiety, probably due to the lower work demands in the studied low COVID-19 burden setting compared to regions overwhelmed by the health crisis. Quite unexpectedly since receiving support from family embodies an efficient strategy to cope with stressful constellations [39], feeling supported by family was found to be positively correlated with anxiety levels in the present study. This finding does not indicate a causal relationship. It possibly mirrors both the sensitization of families to the needs of hospital workforces for support during the COVID19 crisis and the easy access of hospital workforces to this mental support source in the studied setting of low COVID19 burden (e.g. no work overload, enough time to spend with family). The detected inverse association between age and anxiety is in line with past reports [17, 41, 42] and may be explained by more efficient use of problem-solving-, cognitive restructuring- and express emotion coping strategies with advancing age [43]. This advantage of advancing age might be useful in implementing and fostering (one-to-one) peer support interventions, which are on the increase worldwide [44-46], to enhance efficacy of hospital workforces in coping with the COVID-19 crisis, although even such interventions are not immune to the detrimental effects of the pandemic crisis and the difficulties related to its management (e.g. social distancing, reliance on communication via internet) [47].

Depression symptom severity positively correlated with intrusion symptoms and family support, whilst it was inversely influenced by preventive behaviours and attitudes towards COVID-19 and crisis duration. Again, the unveiled significant association between intrusion symptoms and depression symptom severity points to the relationship between COVID-19 crisis, as traumatic experience, and depression symptoms. Contrary to anxiety, depression symptoms were not found to be related to avoidance symptoms, possibly due to the relatively loose coupling of these two symptom groups especially in the absence of a major depressive episode [48]. The negative association between time since the diagnosis of the first COVID-19 case in Greece and depression symptoms in hospital workforces may reflect the euphoria feelings that were increasingly prompted, as time passed, by the low COVID-19 burden and the successful management of the health crisis in spring 2020 in Greece [49]. Moreover, the inverse relation between both preventive behaviours and attitudes towards COVID-19 and depressive symptom severity accords with past reports $[16,36]$ and underscores the importance of having at your disposal tools to protect yourself and endure adversities. In such a way, feelings of high vulnerability and hopelessness decrease [50]; so does depression risk. The observed trend towards significance of the negative association between worries related to COVID-19 infection and depression symptoms should be treated with caution. It may be a spurious finding and warrants further investigation especially in the light of the previous report on the linkage between higher resilience and lower COVID-19 related worries [51].

The present study has a number of limitations. The representative nature of the study is obviously limited because of the small sample size, the high dropout rates, particularly in the backstage hospital workforce group, and the lack of a strong sampling frame, especially due to data collection also through an online questionnaire, which was mainly distributed through social media [26]. Volunteer participants were self-selected and so the generalizability of the results remains to be determined. Moreover, owing to the cross-sectional study design, no conclusions with regard to causality can be drawn. Anxiety and depression symptoms were assessed with validated and reliable, albeit different instruments in online survey participants and hospital workforces. Nonetheless, HADS, GAD-7, PHQ-9 do capture anxiety and/or depression with comparable efficacy [52] and they do not classify depression or anxiety severity symptoms independently, meaning that they obtain concordant severity classifications [53]. Considering anxiety and depression symptom severity in the analyses and not HADS, GAD-7 and PHQ-9 raw data was a pragmatic strategy which enabled the comparison of anxiety and depression symptoms between hospital workforces and general population online survey participants. Enriching the study sample and design with participants of a convenience general population online survey [26], conducted during the first COVID-19 confinement period in Greece, resulted in a relatively long time 
frame of data collection. The detected significant differences in the time since the diagnosis of the first COVID-19 case in Greece between hospital workforces and online survey participants may be considered as another shortcoming of the present study. It is unlikely that this difference has biased our findings, since regression analyses, which included time since the detection of the first COVID-19 as independent variable, did unveil significant associations between anxiety symptom severity and serving as frontline- or backstage hospital employee (Table 4). It should also be underscored that online survey data collection took place during confinement, a highly distressing period of time, during which the risk for adverse mental health outcomes in general population reaches its zenith [41]. Nonetheless, the differences in anxiety and depression symptom severity between online survey participants and hospital workforces reached statistical significance, highlighting the heavy mental burden of the latter. Furthermore, factors that have been shown to affect the development of anxiety and depression symptoms during the pandemic such as history of mental disorder, somatic morbidities, personality structure, loneliness feelings, lower household income, financial loss $[17,19]$ were not considered in the present study.

Further studies with larger cohorts and longitudinal design are warranted before final conclusions with regard to the relationship between anxiety- and depression symptoms in hospital workforces and their reaction to COVID19 crisis in regions with low COVID-19 burden can be drawn. The observations of the present study clearly highlight the significant effects of intrusion, avoidance, age, family support, preventive behaviours, attitudes and worries linked to the COVID-19 crisis on such symptoms and shed light on factors pertaining to high vulnerability to anxiety and depression symptoms. Our findings serve as an alarming voice for the support needs of hospital employees during the COVID-19 pandemic independently of local COVID-19 burden. Organizational adjustments (e.g. shortened shifts, staff involvement in organisational decisions), effective (online) listening and communication [54], easily accessible professional support (e.g. web- or telephone based hotlines, hospital-based teams providing counselling on handling stress) [39] and models such as the structured forum Schwartz rounds [55] and (one-to-one) peer support [44-47] may contribute to the alleviation of the distress of hospital workforces due to the COVID-19 crisis.

Funding This research received no specific grant from any funding agency, commercial or not-for-profit sectors.

\section{Declarations}

Conflict of interest Authors declare no conflict of interest.

\section{References}

1. Forte G, Favieri F, Tambelli R et al (2020) COVID-19 pandemic in the Italian population: validation of a post-traumatic stress disorder questionnaire and prevalence of PTSD symptomatology. Int J Environ Res Public Health. https://doi.org/10.3390/ijerph1711 4151

2. Hu B, Guo H, Zhou P et al (2020) Characteristics of SARS-CoV-2 and COVID-19. Nat Rev Microbiol. https://doi.org/10.1038/ s41579-020-00459-7

3. Anastasopoulou S, Athanasia M (2020) The biology of SARSCoV-2 and the ensuing COVID-19. Achaiki Iatriki 39:29-35

4. Mukaetova-Ladinska EB, Kronenberg G (2020) Psychological and neuropsychiatric implications of COVID-19. Eur Arch Psychiatry Clin Neurosci. https://doi.org/10.1007/s00406-020-01210-2

5. McCracken LM, Badinlou F, Buhrman M et al (2020) Psychological impact of COVID-19 in the Swedish population: depression, anxiety, and insomnia and their associations to risk and vulnerability factors. Eur Psychiatry 63:e81. https://doi.org/10.1192/j. eurpsy.2020.81

6. Seghi F, Barbini B, Franchini L et al (2020) The challenge of mental health during Covid-19 outbreak: experience from metropolitan area of Milan. Eur Arch Psychiatry Clin Neurosci. https:// doi.org/10.1007/s00406-020-01154-7

7. Chew NWS, Lee GKH, Tan BYQ et al (2020) A multinational, multicentre study on the psychological outcomes and associated physical symptoms amongst healthcare workers during COVID19 outbreak. Brain Behav Immun. https://doi.org/10.1016/j.bbi. 2020.04.049

8. Arpacioglu S, Gurler M, Cakiroglu S (2020) Secondary traumatization outcomes and associated factors among the health care workers exposed to the COVID-19. Int J Soc Psychiatry. https:// doi.org/10.1177/0020764020940742

9. Lai J, Ma S, Wang Y et al (2020) Factors associated with mental health outcomes among health care workers exposed to coronavirus disease 2019. JAMA Netw Open 3:e203976. https://doi.org/ 10.1001/jamanetworkopen.2020.3976

10. Liang Y, Chen M, Zheng X et al (2020) The psychological status of frontline health workers confronting COVID-19 after local cases eradication in Zhuhai, Southern China. Eur Arch Psychiatry Clin Neurosci. https://doi.org/10.1007/s00406-020-01204-0

11. Kramer V, Papazova I, Thoma A et al (2020) Subjective burden and perspectives of German healthcare workers during the COVID-19 pandemic. Eur Arch Psychiatry Clin Neurosci. https:// doi.org/10.1007/s00406-020-01183-2

12. Greenberg N (2020) Mental health of health-care workers in the COVID-19 era. Nat Rev Nephrol 16:425-426. https://doi.org/10. 1038/s41581-020-0314-5

13. Shale S (2020) Moral injury and the COVID-19 pandemic: reframing what it is, who it affects and how care leaders can manage it. BMJ Leader. https://doi.org/10.1136/leader-2020-000295

14. Zhizhong W, Koenig HG, Yan T et al (2020) Psychometric properties of the moral injury symptom scale among Chinese health professionals during the COVID-19 pandemic. BMC Psychiatry 20:556. https://doi.org/10.1186/s12888-020-02954-w

15. McFee RB (2020) COVID-19 mental health considerations for health care workers and patients: a brief overview. Dis Mon 66:101061. https://doi.org/10.1016/j.disamonth.2020.101061

16. Gold JA (2020) Covid-19: adverse mental health outcomes for healthcare workers. BMJ 369:m1815. https://doi.org/10.1136/bmj. m1815

17. Braquehais MD, Vargas-Cáceres S, Gómez-Durán E et al (2020) The impact of the COVID-19 pandemic on the mental health of healthcare professionals. QJM. https://doi.org/10.1093/qjmed/ hcaa207 
18. Tan BYQ, Chew NWS, Lee GKH et al (2020) Psychological impact of the COVID-19 pandemic on health care workers in Singapore. Ann Intern Med 173:317-320. https://doi.org/10.7326/ M20-1083

19. Young KP, Kolcz DL, O'Sullivan DM et al (2020) Health care workers' mental health and quality of life during COVID-19: results from a mid-pandemic, national survey. Psychiatr Serv. https://doi.org/10.1176/appi.ps.202000424

20. Abdessater M, Rouprêt M, Misrai V et al (2020) COVID-19 outbreak situation and its psychological impact among surgeons in training in France. World J Urol. https://doi.org/10.1007/ s00345-020-03207-x

21. Spoorthy MS, Pratapa SK, Mahant S (2020) Mental health problems faced by healthcare workers due to the COVID-19 pandemic - a review. Asian J Psychiatr 51:102119. https://doi.org/ 10.1016/j.ajp.2020.102119

22. Wang W, Song W, Xia Z et al (2020) Sleep disturbance and psychological profiles of medical staff and non-medical staff during the early outbreak of COVID-19 in Hubei Province. China Front Psychiatry 11:733. https://doi.org/10.3389/fpsyt.2020.00733

23. Yang J, Chen X, Deng X et al (2020) Disease burden and clinical severity of the first pandemic wave of COVID-19 in Wuhan. China Nat Commun 11:5411. https://doi.org/10.1038/ s41467-020-19238-2

24. Hale T, Angrist N, Cameron-Blake E, Hallas L, Kira B, Majumdar S, Petherick A, Phillips T, Tatlow H, Webster S (2020) Oxford COVID-19 government response tracker, Blavatnik School of Government. www.bsg.ox.ac.uk/covidtracker. Accessed 10 Dec 2020

25. Peppou LE, Economou M, Skali T et al (2020) From economic crisis to the COVID-19 pandemic crisis: evidence from a mental health helpline in Greece. Eur Arch Psychiatry Clin Neurosci. https://doi.org/10.1007/s00406-020-01165-4

26. Karaivazoglou K, Konstantopoulou G, Kalogeropoulou M et al (2021) Psychological distress in the Greek general population during the first COVID-19 lockdown. BJPsych Open 7:e59. https:// doi.org/10.1192/bjo.2021.17

27. Mystakidou K, Tsilika E, Parpa E et al (2007) Psychometric properties of the impact of event scale in Greek cancer patients. J Pain Symptom Manage 33:454-461. https://doi.org/10.1016/j.jpain symman.2006.09.023

28. Hyphantis T, Kotsis K, Voulgari PV et al (2011) Diagnostic accuracy, internal consistency, and convergent validity of the Greek version of the patient health questionnaire 9 in diagnosing depression in rheumatologic disorders. Arthritis Care Res (Hoboken) 63:1313-1321. https://doi.org/10.1002/acr.20505

29. Gilbody S, Richards D, Brealey S et al (2007) Screening for depression in medical settings with the Patient Health Questionnaire (PHQ): a diagnostic meta-analysis. J Gen Intern Med 22:1596-1602. https://doi.org/10.1007/s11606-007-0333-y

30. Sun Y, Fu Z, Bo Q et al (2020) The reliability and validity of PHQ-9 in patients with major depressive disorder in psychiatric hospital. BMC Psychiatry 20:474. https://doi.org/10.1186/ s12888-020-02885-6

31. Kroenke K, Spitzer RL, Williams JB (2001) The PHQ-9: validity of a brief depression severity measure. J Gen Intern Med 16:606613. https://doi.org/10.1046/j.1525-1497.2001.016009606.x

32. Li X, Tian J, Xu Q (2020) The associated factors of anxiety and depressive symptoms in COVID-19 patients hospitalized in Wuhan, China. Psychiatr Q. https://doi.org/10.1007/ s11126-020-09865-9

33. Michopoulos I, Douzenis A, Kalkavoura C et al (2008) Hospital anxiety and depression scale (HADS): validation in a Greek general hospital sample. Ann Gen Psychiatry 7:4. https://doi.org/10. $1186 / 1744-859$ X-7-4
34. Parlapani E, Holeva V, Voitsidis P et al (2020) Psychological and behavioral responses to the COVID-19 pandemic in Greece. Front Psychiatry 11:821. https://doi.org/10.3389/fpsyt.2020.00821

35. Johnson SU, Ulvenes PG, Øktedalen T et al (2019) Psychometric properties of the general anxiety disorder 7-item (GAD-7) scale in a heterogeneous psychiatric sample. Front Psychol 10:1713. https://doi.org/10.3389/fpsyg.2019.01713

36. Alizadeh A, Khankeh HR, Barati M et al (2020) Psychological distress among Iranian health-care providers exposed to coronavirus disease 2019 (COVID-19): a qualitative study. BMC Psychiatry 20:494. https://doi.org/10.1186/s12888-020-02889-2

37. Cabarkapa S, King JA, Ng CH (2020) The psychiatric impact of COVID-19 on healthcare workers. Aust J Gen Pract 49:791-795. https://doi.org/10.31128/AJGP-07-20-5531

38. Hossain F, Clatty A (2020) Self-care strategies in response to nurses' moral injury during COVID-19 pandemic. Nurs Ethics. https://doi.org/10.1177/0969733020961825

39. Muller AE, Hafstad EV, Himmels JPW et al (2020) The mental health impact of the covid-19 pandemic on healthcare workers, and interventions to help them: a rapid systematic review. Psychiatry Res 293:113441. https://doi.org/10.1016/j.psychres.2020. 113441

40. Konstantopoulou G, Iliou T, Karaivazoglou K et al (2020) Associations between (sub) clinical stress- and anxiety symptoms in mentally healthy individuals and in major depression: a crosssectional clinical study. BMC Psychiatry 20:428. https://doi.org/ 10.1186/s12888-020-02836-1

41. Henssler J, Stock F, van Bohemen J et al (2020) Mental health effects of infection containment strategies: quarantine and isolation-a systematic review and meta-analysis. Eur Arch Psychiatry Clin Neurosci. https://doi.org/10.1007/s00406-020-01196-X

42. Soares JJF, Grossi G, Sundin O (2007) Burnout among women: associations with demographic/socio-economic, work, life-style and health factors. Arch Womens Ment Health 10:61-71. https:// doi.org/10.1007/s00737-007-0170-3

43. Monteiro NM, Balogun SK, Oratile KN (2014) Managing stress: the influence of gender, age and emotion regulation on coping among university students in Botswana. Int J Adolesc Youth 19:153-173. https://doi.org/10.1080/02673843.2014.908784

44. White S, Foster R, Marks J et al (2020) The effectiveness of oneto-one peer support in mental health services: a systematic review and meta-analysis. BMC Psychiatry 20:534. https://doi.org/10. 1186/s12888-020-02923-3

45. Puschner B, Repper J, Mahlke C et al (2019) Using peer support in developing empowering mental health services (UPSIDES): background, rationale and methodology. Ann Glob Health. https:// doi.org/10.5334/aogh.2435

46. Burke EM, Pyle M, Machin K et al (2018) Providing mental health peer support 1: a Delphi study to develop consensus on the essential components, costs, benefits, barriers and facilitators. Int J Soc Psychiatry 64:799-812. https://doi.org/10.1177/0020764018 810299

47. Mpango R, Kalha J, Shamba D et al (2020) Challenges to peer support in low- and middle-income countries during COVID-19. Global Health 16:90. https://doi.org/10.1186/s12992-020-00622-y

48. Ottenbreit ND, Dobson KS, Quigley L (2014) An examination of avoidance in major depression in comparison to social anxiety disorder. Behav Res Ther 56:82-90. https://doi.org/10.1016/j.brat. 2014.03.005

49. Moris D, Schizas D (2020) Lockdown during COVID-19: the Greek success. In Vivo 34:1695-1699. https://doi.org/10.21873/ invivo. 11963

50. Liu RT, Kleiman EM, Nestor BA et al (2015) The hopelessness theory of depression: a quarter century in review. Clin Psychol (New York) 22:345-365. https://doi.org/10.1111/cpsp.12125 
51. Barzilay R, Moore TM, Greenberg DM et al (2020) Resilience, COVID-19-related stress, anxiety and depression during the pandemic in a large population enriched for healthcare providers. Transl Psychiatry 10:291. https://doi.org/10.1038/ s41398-020-00982-4

52. Marrie RA, Zhang L, Lix LM et al (2018) The validity and reliability of screening measures for depression and anxiety disorders in multiple sclerosis. Mult Scler Relat Disord 20:9-15. https://doi. org/10.1016/j.msard.2017.12.007

53. Nyongesa MK, Mwangi P, Koot HM et al (2020) The reliability, validity and factorial structure of the Swahili version of the 7-item generalized anxiety disorder scale (GAD-7) among adults living with HIV from Kilifi. Kenya Ann Gen Psychiatry. https://doi.org/ 10.1186/s12991-020-00312-4

54. Itzchakov G, Grau J (2020) High-quality listening in the age of COVID-19. Org Dyn. https://doi.org/10.1016/j.orgdyn.2020. 100820

55. Gleeson D, Arwyn-Jones J, Awan M et al (2020) Medical student Schwartz rounds: a powerful medium for medical student reflective practice. Adv Med Educ Pract 11:775-780. https://doi.org/ 10.2147/AMEP.S273181 